\title{
Limiares auditivos em frequências altas e emissões otoacústicas em pacientes com anemia falciforme
}

\author{
High frequencies thresholds and otoacoustics emissions in patients with sickle cell anemia \\ Umbrales auditivos y emisiones otoacústicas en pacientes con anemia falciforme
}

Liese Loureiro Weigert

ORCID: https://orcid.org/ 000-0002-8898-1442 Hospital de Clínicas de Porto Alegre, Brasil

E-mail: 1weigert@hcpa.edu.br

Adriane Ribeiro Teixeira

ORCID: https://orcid.org/ 000-0003-4242-1666 Universidade Federal do Rio Grande do Sul, Brasil Hospital de Clínicas de Porto Alegre, Brasil

E-mail: adriane.teixeira@gmail.br

Lúcia Bencke Geyer

ORCID: https://orcid.org/ 000-0001-7474-318X Hospital de Clínicas de Porto Alegre, Brasil

E-mail: lbgeyer@hcpa.edu.br

Christina Matzembacher Bittar

ORCID: https://orcid.org/ 000-0003-2839-9677 Hospital de Clínicas de Porto Alegre, Brasil E-mail: cbittar@hcpa.edu.br

Claudia Schweiger

ORCID: https://orcid.org/ 000-0002-3898-0723 Hospital de Clínicas de Porto Alegre, Brasil E-mail: causch@hcpa.edu.br

Bruna Macagnin Seimetz

ORCID: https://orcid.org/ 000-0001-6391-8183 Universidade Federal do Rio Grande do Sul, Brasil E-mail: bruna.seimetz@gmail.com

Nathany Lima Ruschel

ORCID: https://orcid.org/ 000-0002-6354-5425 Universidade Federal do Rio Grande do Sul, Brasil E-mail: nathanyruschel@ hotmail.com

Joziane Moraes Lima

ORCID: https://orcid.org/ 000-0003-4698-2989 Hospital de Clínicas de Porto Alegre, Brasil E-mail: jozimoraeslima@gmail.com

Sérgio Saldanha Menna Barreto

ORCID: https://orcid.org/ 000-0003-2000-2866 Universidade Federal do Rio Grande do Sul, Brasil Hospital de Clínicas de Porto Alegre, Brasil E-mail: smenna@terra.com.br

\begin{abstract}
Resumo
Objetivo: Verificar limiares auditivos em frequências convencionais e altas frequências e presença de emissões otoacústicas por produto de distorção em pacientes falcêmicos, analisando a variável faixa etária. Método: Coorte observacional, prospectivo, analítico, de fins diagnósticos. Realizada audiometria convencional, audiometria em frequências altas (AAF) e emissões otoacústicas por produto de distorção (EOAPD) em pacientes portadores da doença. Resultados: Foram avaliados 38 pacientes, sendo $16(42,1 \%)$ na faixa etária de 7 a 20 anos de idade e $22(57,9$ $\%$ ), de 21 a 35 anos. A alteração auditiva na audiometria convencional foi de 6,3\% na faixa etária de 7 a 20 anos e 13 , $6 \%$, entre 21 e 35 anos. Não houve diferença estatisticamente significativa entre as faixas etárias ( $\mathrm{p}=0,624)$. Na AAF, alteração em $25 \%$ das crianças e adolescentes e em $68,2 \%$ dos adultos. Os adultos apresentaram maior perda auditiva ( $\mathrm{p}=0,021)$. Não houve diferença significativa na comparação das medidas de relação sinal/ruído das EOAPD entre as orelhas, tanto na amostra total quanto por faixa etária $(p>0,05)$. Não houve associação significativa entre os resultados das alterações das EOAPD e a perda auditiva em frequências ultra-altas tanto nas crianças e adolescentes $(\mathrm{p}=0,250)$ quanto nos adultos $(\mathrm{p}=0,121)$. Na comparação entre as sensibilidades dos exames AAF e EOAPD, houve diferença significativa entre eles somente nos adultos $(\mathrm{p}=0,004)$. Conclusões: Os adultos com maior duração da exposição à doença apresentaram elevação dos limiares em AAF, bem como alteração das EOAPD.
\end{abstract}


Palavras-chave: Doença falciforme; Audição; Audiometria; Perda auditiva.

\begin{abstract}
Objective: To verify auditory thresholds at conventional and high frequencies and the presence of distortion product otoacoustic emissions in sickle cell patients, analyzing the age group variable. Method: Observational, prospective, analytical cohort for diagnostic purposes. Conventional audiometry, high frequency audiometry and distortion product otoacoustic emissions were performed in patients with the disease. Results: 38 patients were evaluated, $16(42.1 \%)$ in the age group from 7 to 20 years old and 22 (57.9\%), from 21 to 35 years old. Hearing impairment in conventional audiometry was $6.3 \%$ in the age group of 7 to 20 years and 13, 6\%, between 21 and 35 years. There was no statistically significant difference between the age groups $(\mathrm{p}=0.624)$. In high frequency audiometry, changes in $25 \%$ of children and adolescents and in $68.2 \%$ of adults. Adults had greater hearing loss $(\mathrm{p}=0.021)$. There was no significant difference when comparing the distortion product otoacoustic emissions signal / noise ratio measurements between the ears, both in the total sample and by age group ( $p>0.05)$. There was no significant association between the results of the distortion product otoacoustic emissions changes and hearing loss at ultra-high frequencies both in children and adolescents $(\mathrm{p}=0.250)$ and in adults $(\mathrm{p}=0.121)$. When comparing the sensitivity of the high frequency audiometry and distortion product otoacoustic emissions exams, there was a significant difference between them only in adults $(\mathrm{p}=0.004)$. Conclusions: Adults with a longer duration of exposure to the disease showed increased thresholds in high frequency audiometry, as well as changes in distortion product otoacoustic emissions.
\end{abstract}

Keywords: Anemia sickle cell; Hearing; Audiometry; Hearing loss.

\title{
Resumen
}

Objetivo: verificar los umbrales auditivos a frecuencias convencionales y altas y la presencia de otoemisiones acústicas producto de distorsión en pacientes con anemia falciforme, analizando la variable grupo de edad. Método: Cohorte observacional, prospectiva, analítica con fines diagnósticos. Se realizó audiometría convencional, audiometría de alta frecuencia (AAF) y otoemisiones acústicas de productos de distorsión (DPOAE) en pacientes con la enfermedad. Resultados: se evaluaron 38 pacientes, $16(42,1 \%)$ en el grupo de edad de 7 a 20 años y 22 (57,9\%), de 21 a 35 años. La discapacidad auditiva en audiometría convencional fue del 6,3\% en el grupo de edad de 7 a 20 años y del 13,6\%, entre 21 y 35 años. No hubo diferencia estadísticamente significativa entre los grupos de edad $(\mathrm{p}=0,624)$. En AAF, cambios en el $25 \%$ de los niños y adolescentes y en el 68,2\% de los adultos. Los adultos tenían una mayor pérdida auditiva $(\mathrm{p}=0,021)$. No hubo diferencia significativa al comparar las medidas de la relación señal / ruido EOAPD entre los oídos, tanto en la muestra total como por grupo de edad ( $p>0.05)$. No hubo asociación significativa entre los resultados de los cambios de DPOAE y la pérdida de audición en frecuencias ultra altas tanto en niños y adolescentes $(\mathrm{p}=0,250)$ como en adultos $(\mathrm{p}=0,121)$. Al comparar las sensibilidades de los exámenes AAF y DPOAE, hubo una diferencia significativa entre ellos solo en adultos $(\mathrm{p}=0,004)$. Conclusiones: Los adultos con una mayor duración de exposición a la enfermedad mostraron umbrales aumentados en AAF, así como cambios en DPOAE.

Palabras clave: Anemia de celulas falciformes; Audición; Audiometry; Pérdida auditiva.

\section{Introdução}

A anemia falciforme (AF) é a doença hereditária mais prevalente no Brasil, ocorrendo predominantemente entre afrodescendentes. Trata-se de uma hemoglobinopatia hereditária causada por alterações genéticas com predominância da hemoglobina S, a qual confere às hemácias a forma de foice, que deu origem ao nome falciforme (Burch-Sims \& Matlock, 2005; Silva, Nova \& Lucena, 2012). A forma anômala da hemácia, causada pelo decréscimo de oxigênio, impossibilita a circulação adequada o que gera obstrução do fluxo sanguíneo e consequentemente várias manifestações clínicas, sendo uma delas a vaso-oclusão (Burch-Sims \& Matlock, 2005; Farrel, Landry, Yee, Leu \& Goudy, 2019; Lago et al., 2018; Olajuyin, Olatunya, Adegbiji, Oyenibi \& Faboya, 2018; Sarac, Boke \& Okuyucu, 2018). Os fenômenos de vaso-oclusão de repetição e a hemólise intravascular crônica determinam fenômenos isquêmicos agudos e crônicos que acabam determinando a vasculopatia crônica com prejuízo especialmente da microcirculação (Potoka \& Gladwin, 2015).

Levando-se em consideração o comprometimento do fluxo sanguíneo, que pode gerar inúmeras complicações em órgãos, sistemas e aparelhos. A literatura também aponta relação da doença com a perda auditiva, causada em função da anóxia no órgão de Corti (Burch-Sims \& Matlock, 2005; Farrel, Landry, Yee, Leu \& Goudy, 2019; Olajuyin, Olatunya, Adegbiji, Oyenibi \& Faboya, 2018; Silva, Nova \& Lucena, 2012; Stuart \& Preast, 2012). O sistema auditivo tem irrigação vascular rica, principalmente na estria vascular, situada no órgão de Corti, dentro da cóclea. A alta taxa de consumo de 
oxigênio e a pobre capacidade de metabolismo anaeróbico nesta região torna a orelha interna sensível à anóxia/hipóxia (Farrel, Landry, Yee, Leu \& Goudy, 2019; Lago et al., 2018; Sarac, Boke \& Okuyucu, 2018; Stuart \& Preast, 2012).

A perda auditiva neurossensorial (PANS), causada pelo vaso-oclusão dos vasos sanguíneos labirínticos, pode resultar em hemorragia labiríntica e labirintite ossificante. A cóclea por ter sua irrigação realizada por apenas uma artéria terminal, a artéria labiríntica, torna o ouvido interno mais sensível a alterações da circulação sanguínea. A oclusão desta artéria de forma total e permanente poderia justificar a perda auditiva, que na maioria nos casos ocorre de forma súbita, mas não seria suficiente para explicar o porquê da recuperação audiométrica frequente, nem a ausência de fibrose e ossificação coclear, em estudos post-mortem de indivíduos que sofreram surdez súbita (Lago et al., 2018; Oliveira et al., 2009). Surgiu assim a noção de hipoperfusão sanguínea dentro da teoria vascular, como causa da disfunção coclear. Ocorreria devido a fenômenos de obstrução parcial ou temporária, em alternativa por hiperviscosidade sanguínea, a qual levaria a uma redução da oxigenação da cóclea (Rodrigues, 2017).

A literatura aponta alguns estudos com avaliações audiológicas e portadores de AF, inclusive alguns estudos com emissões otoacústicas (EAO) e audiometria de altas frequências (AAF), mas ainda há carência de pesquisas, sendo que as existentes não apresentam concordância em seus resultados. Cada amostra, com diferentes faixas etárias e avaliações, demostram resultados específicos, como valores aumentados nos limiares auditivos (Sarac, Boke \& Okuyucu, 2018), ausência de resultados estatisticamente significativos na comparação de resultados audiológicos em portadores de AF e grupo controle (Kegele et al., 2015), baixa porcentagem de perda auditiva em crianças com AF (Farrel, Landry, Yee, Leu \& Goudy, 2019) e maior prevalência de perda auditiva de grau leve em sujeitos com AF (Olajuyin, Olatunya, Adegbiji, Oyenibi \& Faboya, 2018).

A pesquisa de EOA trata-se de uma avaliação sensível à detecção precoce ou subclínica de danos cocleares ainda imperceptíveis em audiometria. As EOA atuam como indicador da condição fisiológica coclear, através da contração das células ciliadas externas (CC). O mau funcionamento das Células Ciliadas Externas (CCEs), amplificadores cocleares, possivelmente seja o fator mais crítico que explica a deterioração das emissões otoacústicas (Psaltakos et al, 2013). Os decréscimos na amplitude e na área dinâmica das respostas como também a perda de resposta das EOA por produto de distorção (OEAPD) podem ser percebidas quando há mudança na função das CCE. As EOAPD são consideradas mais vantajosas para avaliar a função coclear, pois permitem verificar o crescimento da resposta de acordo com a variação dos níveis dos estímulos sonoros apresentados (Silva, Fiorini \& Azevedo, 2017).

A investigação de possíveis comprometimentos auditivos em indivíduos portadores de AF se faz relevante devido à alta prevalência da doença no Brasil e da probabilidade de atingir uma parcela maior da população, em função da miscigenação do país. O aumento do número de pesquisas a respeito do assunto, possibilita a conscientização da população em relação à importância da realização de avaliação audiológica precoce, como EOA e audiometria tonal liminar (ATL) e AAF, evitando assim, a privação dos estímulos auditivos, principalmente nos primeiros anos de vida, período fundamental para aquisição e desenvolvimento da linguagem.

Assim, o objetivo desta pesquisa é avaliar os limiares auditivos em frequências convencionais e altas frequências e as emissões otoacústicas por produto de distorção em pacientes diagnosticados com anemia falciforme.

\section{Metodologia}

O projeto que originou esta pesquisa foi analisado e aprovado pelo Comitê de Ética em Pesquisa da instituição onde o estudo foi realizado, com número de protocolo de aprovação 11-0245.

O tipo de estudo realizado foi quantitativo, prospectivo e descritivo (não experimental ou ex post facto). Assim, buscou-se estudar as relações entre as variáveis, mas sem manipulá-las, verificando somente as relações entre elas em situações já existentes (Köche, 2011). 
Todos os participantes da pesquisa foram provenientes de ambulatório especializado em hematologia da instituição, apresentavam diagnóstico de anemia falciforme e assinaram o Termo de Consentimento Livre e Esclarecido (pacientes ou responsáveis).

Foram incluídos no estudo pacientes com diagnóstico de anemia falciforme, com idades entre 07 e 35 anos, realizando o tratamento definido pelos médicos responsáveis. Foram excluídos pacientes com histórico de exposição a ruído de intensidade elevada, incluindo fones de ouvido nas 14 horas antecedentes ao exame; pacientes com comorbidade aguda no momento do exame ou com alteração na orelha média.

Após análise dos critérios de inclusão e exclusão, os pacientes foram avaliados por meio de meatoscopia. Os que apresentavam cera obstrutiva no conduto auditivo foram encaminhados para limpeza otológica com os médicos otorrinolaringologistas do hospital, permitindo a realização das etapas posteriores do estudo.

$\mathrm{Na}$ sequência foi feita a avaliação auditiva, sendo que todos os exames foram realizados pela mesma fonoaudióloga, devidamente familiarizada e treinada com os equipamentos usados na pesquisa, seguindo o mesmo protocolo.

A ATL e AAF foram realizadas em cabina acusticamente tratada, utilizando-se o audiômetro Siemens Unity PC (Alemanha), calibrado em dB NA de acordo com a norma ANSI s 3.6-1989, com fones aéreo HDA 200 e ósseo B-71. A audiometria tonal por via aérea foi realizada nas frequências de $125 \mathrm{~Hz}, 250 \mathrm{~Hz}, 500 \mathrm{~Hz}, 750 \mathrm{~Hz}, 1.000 \mathrm{~Hz}, 1.500 \mathrm{~Hz}, 2.000$ Hz, $3.000 \mathrm{~Hz}, 4.000 \mathrm{~Hz}, 6.000 \mathrm{~Hz}, 8.000 \mathrm{~Hz}, 9.000 \mathrm{~Hz}, 10.500 \mathrm{~Hz}, 11.200 \mathrm{~Hz}, 12.500 \mathrm{~Hz}, 14.000 \mathrm{~Hz}$ e $16.000 \mathrm{~Hz}$. Por via óssea em $500 \mathrm{~Hz}, 750 \mathrm{~Hz}, 1.000 \mathrm{~Hz}, 1.500 \mathrm{~Hz}, 2.000 \mathrm{~Hz}, 3.000 \mathrm{~Hz}, 4.000 \mathrm{~Hz}$. Foi utilizada a técnica ascendente/descendente, com tom puro modulado (warble tone). O critério de normalidade utilizado para a ATL ( $125 \mathrm{~Hz}$ a $8000 \mathrm{~Hz}$ ) foi a classificação da International Bureau for Audiophonologie (BIAP), na qual a média das frequências de $500 \mathrm{~Hz}, 1.000 \mathrm{~Hz}, 2.000 \mathrm{~Hz}$ e 4.000 Hz menor de 20 dBNA é considerada audição normal (BIAP, 1997)

Para AAF $(9.000 \mathrm{~Hz}$ a $16.000 \mathrm{~Hz})$ foi utilizado como critério de normalidade limiares até $25 \mathrm{~dB}$ NA em uma ou mais frequências, conforme estudos prévios em indivíduos audiologicamente normais (Weigert, Piltcher, Procianoy, Buss \& MennaBarreto, 2013).

Realizou-se imitanciometria a fim de verificar a presença ou não de alteração de orelha média, o que impediria a participação no estudo, porém os dados não foram analisados neste trabalho.

As EOAPD foram realizadas em sala acusticamente tratada. Para a realização do exame colocou-se uma sonda, revestida por uma oliva de látex, no meato acústico externo do paciente. O exame foi realizado nas frequências de $1.000 \mathrm{~Hz}$, $1.400 \mathrm{~Hz}, 2.000 \mathrm{~Hz}, 2.800 \mathrm{~Hz}, 4.000 \mathrm{~Hz}$ e $6.000 \mathrm{~Hz}$ em ambas as orelhas, separadamente. A sonda inserida na orelha do paciente apresentava um estímulo sonoro gerado por dois tons puros de frequências diferentes (F1 e F2) simultaneamente, sendo $F 1=65 \mathrm{~dB}$ e $\mathrm{F} 2=55 \mathrm{~dB}$ e $\mathrm{F} 2 / \mathrm{F} 1=1,22$. O exame foi analisado pelo gráfico PD-grama e considerou-se presença de resposta de amplitudes iguais ou maiores do que 3 dBNPS acima do nível de ruído.

As variáveis quantitativas foram descritas por média e desvio padrão ou mediana e amplitude interquartílica. As variáveis categóricas foram descritas por frequências absolutas e relativas.

Para comparar médias entre os grupos, utilizou-se o teste t-student para amostras independentes. Em caso de assimetria, optou-se pelo teste de Mann-Whitney.

Para avaliar a associação entre as variáveis categóricas, os testes qui-quadrado de Pearson ou exato de Fisher foram aplicados.

A comparação entre as orelhas foi avaliada pelo teste de Wilcoxon.

O nível de significância adotado foi de 5\% e as análises foram realizadas utilizando o programa SPSS versão 21. 


\section{Resultados}

Após a aplicação dos critérios de inclusão e exclusão, a amostra foi constituída por 38 pacientes, divididos em dois grupos. O Grupo 1 foi composto por 16 (42,1\%) indivíduos na faixa etária entre 7 e 20 anos de idade, sendo 11 indivíduos do sexo feminino e 5 do sexo masculino. O Grupo 2 foi formado por $22(57,9 \%)$ pacientes entre 21 e 35 anos, 14 do sexo feminino e 8 do sexo masculino. Não houve diferença significativa entre o número de indivíduos por sexo entre os grupos, bem como entre o tempo de diagnóstico entre os grupos (Tabela 1).

Tabela 1 - Caracterização da amostra.

\begin{tabular}{l|c|c|c}
\hline Variáveis & $\begin{array}{c}\text { Grupo } 1 \\
(7-20 \text { anos }) \\
n=16 ; 42,1 \%\end{array}$ & $\begin{array}{c}\text { Grupo } 2 \\
(21-35 \text { anos }) \\
\mathrm{n}=22 ; 57,9 \%\end{array}$ & $1,000^{*}$ \\
\hline Sexo - n(\%) & $5(31,3)$ & $8(36,4)$ & $14(63,6)$ \\
$\quad$ Masculino & $11(68,8)$ & $28,0 \pm 3,9$ & \\
$\quad$ Feminino & $12,1 \pm 2,9$ & $6,02 \pm 2,76$ & $0,574 * *$ \\
\hline Idade (anos) - média \pm DP & $6,54 \pm 2,65$ & & \\
\hline Tempo de diagnóstico (anos) - média \pm DP & & & \\
\hline
\end{tabular}

Legenda: $\mathrm{n}$ - valores absolutos; \% = valores porcentuais; $\mathrm{DP}=$ desvio padrão; *valores significantes pelo teste qui-quadrado de Pearson; ** valores significantes pelo teste t-student.

Fonte: Autores.

Verificou-se alteração auditiva na audiometria convencional em 6,3\% pacientes do Grupo 1 e 13,6\% do grupo 2. Não houve diferença estatisticamente significativa entre as faixas etárias $(\mathrm{p}=0,624)$. Na AAF, houve alteração auditiva em $25 \%$ dos indivíduos do Grupo 1 e de 68,2\% do Grupo 2, com diferença estatisticamente significativa ( $\mathrm{p}=0,021$ ).

No grupo 1, não houve diferença significativa entre as frequências convencionais e altas ( $\mathrm{p}=0,250$ ), já no grupo 2, foi possível verificar diferença, sendo mais elevadas nas altas $(\mathrm{p}<0,001)$. 
Figura 1 - Presença de perda auditiva entre as faixas etárias nas frequências convencionais $(p=0,624)$ e altas frequências $(\mathrm{p}=0,021)$.

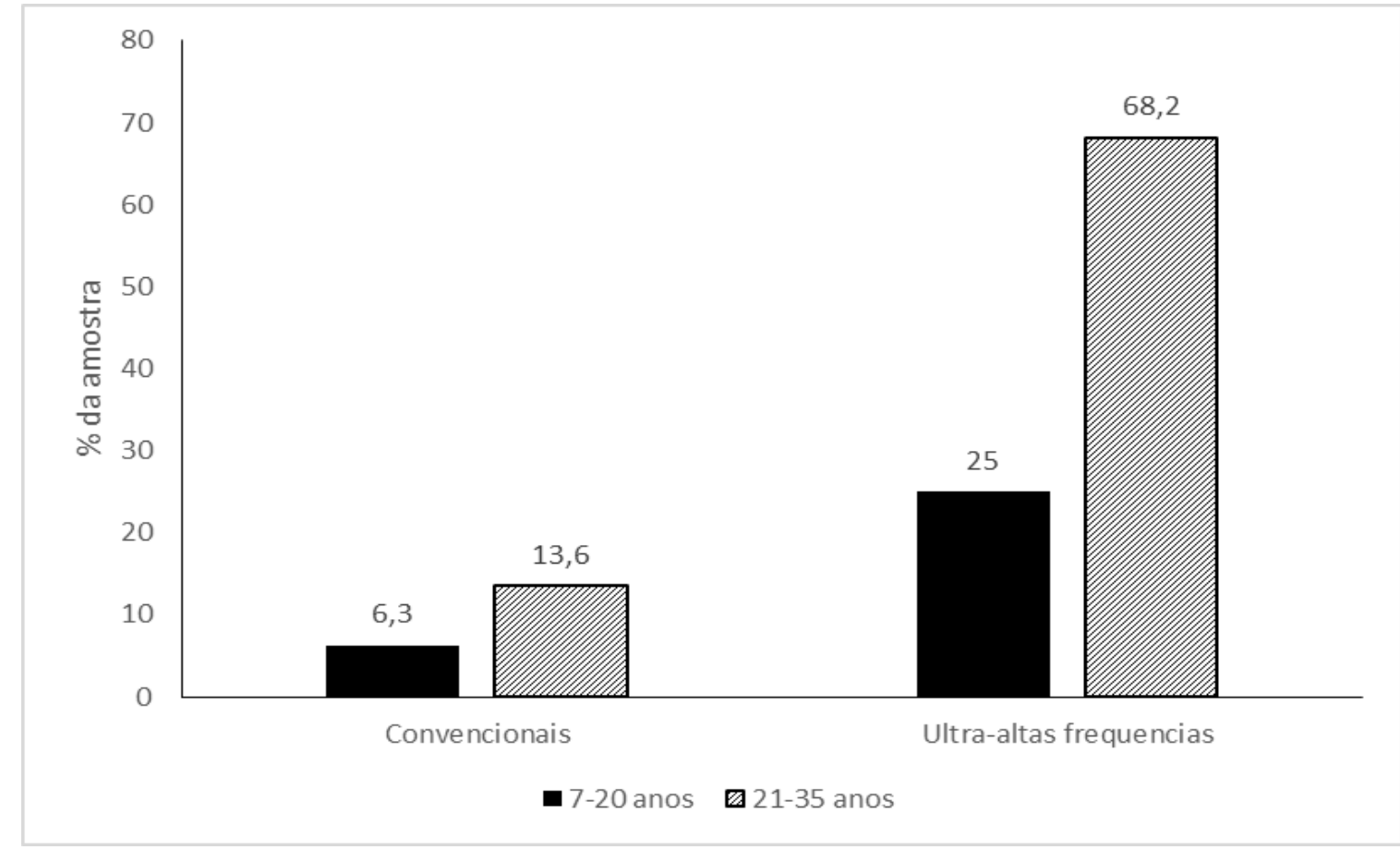

Legenda: \% - valores porcentuais. Fonte: Autores.

Com relação às EOAPD, não houve diferença significativa na relação sinal/ruído (S/R) entre as orelhas tanto na amostra total quanto por faixa etária $(\mathrm{p}>0,05)$. Com isso, realizou-se a comparação das medidas de relação (S/R) entre os grupos. Nessa comparação, as EOAPD foram mais baixas partir de $2.800 \mathrm{~Hz}$ no grupo 2, com diferença estatística (Tabela 2).

Tabela 2 - Comparação das medidas de relação sinal/ruído das EOAPD entre os grupos.

\begin{tabular}{|c|c|c|c|}
\hline Frequências & $\begin{array}{c}\text { Grupo } 1 \\
(7-20 \text { anos }) \\
\text { Md (P25 - P75) }\end{array}$ & $\begin{array}{c}\text { Grupo } 2 \\
(21-35 \text { anos }) \\
\text { Md (P25 - P75) }\end{array}$ & $\mathrm{p}$ \\
\hline $1.000 \mathrm{~Hz}$ & $8,2(0,3$ a 11,4$)$ & $8,7(0,6$ a 12,5$)$ & 0,529 \\
\hline $1.400 \mathrm{~Hz}$ & $15,2(11,5$ a 20,8$)$ & $15,8(9,3$ a 20,7$)$ & 0,942 \\
\hline $2.000 \mathrm{~Hz}$ & $18,1(14,4$ a 23,3$)$ & $15,8(7,2$ a 21,3$)$ & 0,223 \\
\hline $2.800 \mathrm{~Hz}$ & $22,0(16,4$ a 24,5$)$ & $14,1(10,1$ a 19,9$)$ & $0,003 *$ \\
\hline $4.000 \mathrm{~Hz}$ & $21,8(15,6$ a 24,7$)$ & $14,5(9,2$ a 19,7$)$ & $0,019 *$ \\
\hline $6.000 \mathrm{~Hz}$ & $20,5(14,1$ a 24,8$)$ & $10,3(-4,2$ a 17,9$)$ & $0,004 *$ \\
\hline
\end{tabular}

Legenda: p- valores pelo Teste de Mann-Whithney; * valores significantes pelo Teste de Mann-Whithney; Hz - Hertz; $\mathrm{Md}$ - mediana; $\mathrm{P}$ - percentil.

Fonte: Autores.

Foi realizada a comparação das alterações nas EOAPD entre os grupos, de acordo com o critério de presença de emissões através das medidas de relação $\mathrm{S} / \mathrm{R} \geq 3 \mathrm{~dB}$. Encontrou-se diferença significativa entre os grupos quanto às alterações na frequência de $2.800 \mathrm{~Hz}$ na orelha esquerda e $6.000 \mathrm{~Hz}$ na orelha direita, sendo verificados valores maiores no grupo 2 (Tabela 3). 
Tabela 3 - Comparação das alterações nas EOAPD entre os grupos (valores absolutos).

\begin{tabular}{cccc}
\hline Frequências & Grupo 1 & Grupo 2 \\
$(7-20$ anos $)$ & $\mathrm{n}(\%)$ & $(21-35$ anos $)$ & \\
\hline $1.000 \mathrm{~Hz}(\%)$ & 0,469 \\
Direita & $3(18,8)$ & $7(31,8)$ & $0,306^{* *}$ \\
Esquerda & $7(43,8)$ & $5(22,7)$ & 1,000 \\
$1.400 \mathrm{~Hz}$ & & & 0,374 \\
Direita & $2(12,5)$ & $3(13,6)$ & \\
Esquerda & $1(6,3)$ & $4(18,2)$ & 0,624 \\
$2.000 \mathrm{~Hz}$ & $1(6,3)$ & $3(13,6)$ & 0,624 \\
Direita & $1(6,3)$ & $3(13,6)$ & 0,624 \\
Esquerda & & & $0,030^{*}$ \\
$2.800 \mathrm{~Hz}$ & $1(6,3)$ & $3(13,6)$ & \\
Direita & $0(0,0)$ & $6(27,3)$ & 0,426 \\
Esquerda & & & 0,370 \\
$4.000 \mathrm{~Hz}$ & $2(12,5)$ & $6(27,3)$ & 0,374 \\
Direita & $1(6,3)$ & $5(22,7)$ & 0,124 \\
Esquerda & $1(6,3)$ & $11(50,0)$ & $0,012^{* *}$ \\
$6.000 \mathrm{~Hz}$ & $3(18,8)$ & $9(40,9)$ & $0,272^{* *}$ \\
Direita & $1(6,3)$ & $4(18,2)$ & \\
Esquerda & $0(0,0)$ & $4(18,2)$ & \\
Total & & & \\
Direita & & & \\
Esquerda & & & \\
\hline
\end{tabular}

Legenda: $\mathrm{n}$ - valores absolutos; \% - valores porcentuais; $\mathrm{Hz}$ - hertz; *valores significantes pelo teste exato de Fisher; ** valores significantes pelo teste qui-quadrado de Pearson

Fonte: autores.

A associação das alterações das EOAPD com as frequências convencionais, foi realizada somente com grupo 2, pois somente uma criança apresentou perda auditiva, e houve significância estatística $(\mathrm{p}=0,013)$. Na associação entre alterações das EOAPD e perda auditiva em frequências altas, no grupo 1, não houve associação significativa entre os resultados ( $\mathrm{p}=0,250$ ), assim como no grupo 2 ( $\mathrm{p}=0,121)$ (Figura 2$)$. 
Figura 2 - Associação entre os resultados das alterações das emissões otoacústicas por produto de distorção e a perda auditiva em frequências convencionais no grupo $2(\mathrm{p}=0,013)$.

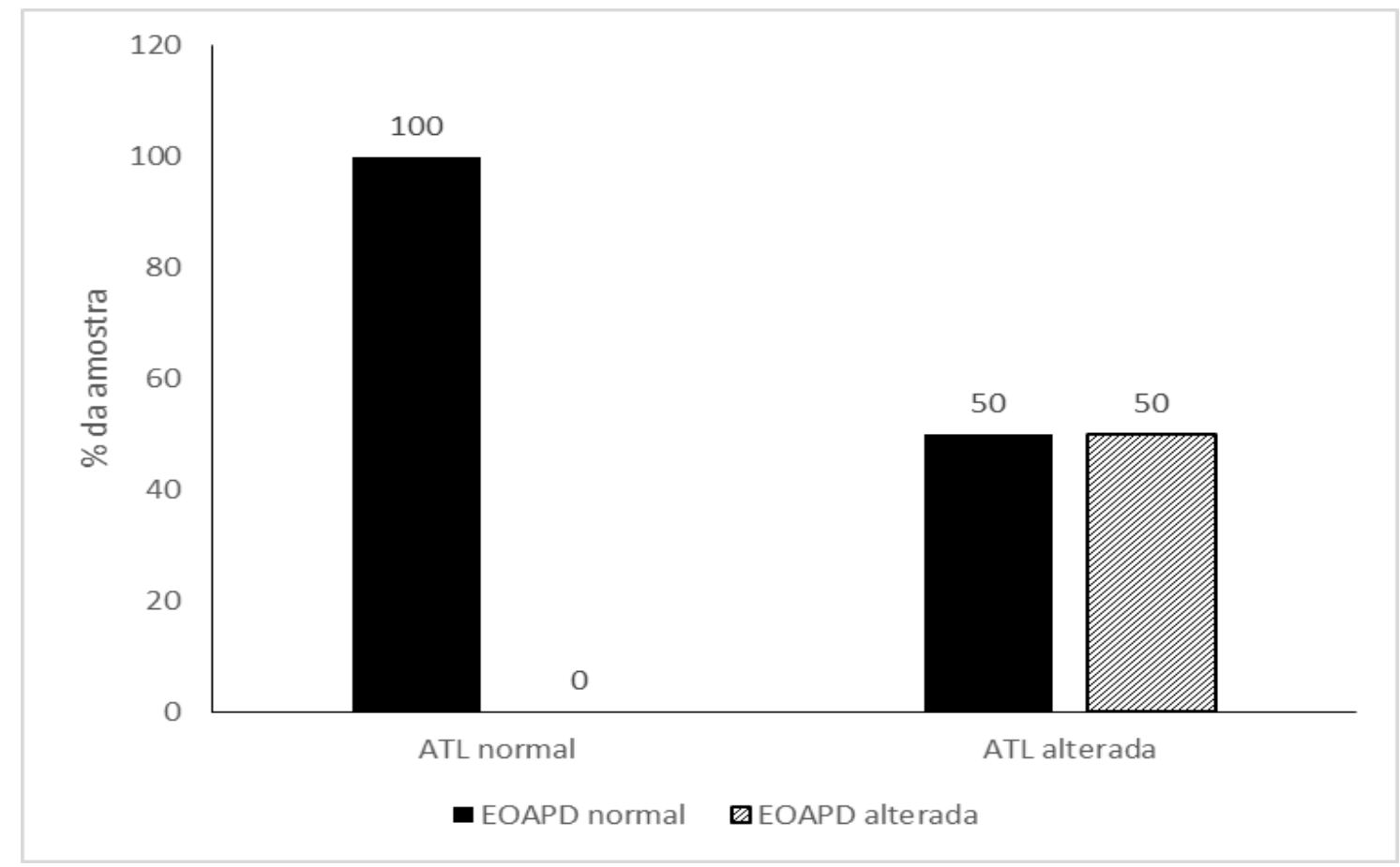

Legenda: \% - valores porcentuais; ATL - audiometria tonal liminar; EOAPD - emissões otoacúticas por produto de distorção Fonte: Autores.

Não houve associação significativa entre os resultados das alterações das EOAPD e perda auditiva em frequências altas, tanto no grupo $1(\mathrm{p}=0,250)$ quanto no grupo $2(\mathrm{p}=0,121)$ (Figura 3 e Figura 4$)$. 
Figura 3 - Associação entre os resultados das alterações das emissões otoacústicas por produto de distorção e a perda auditiva em altas frequências no Grupo 1.

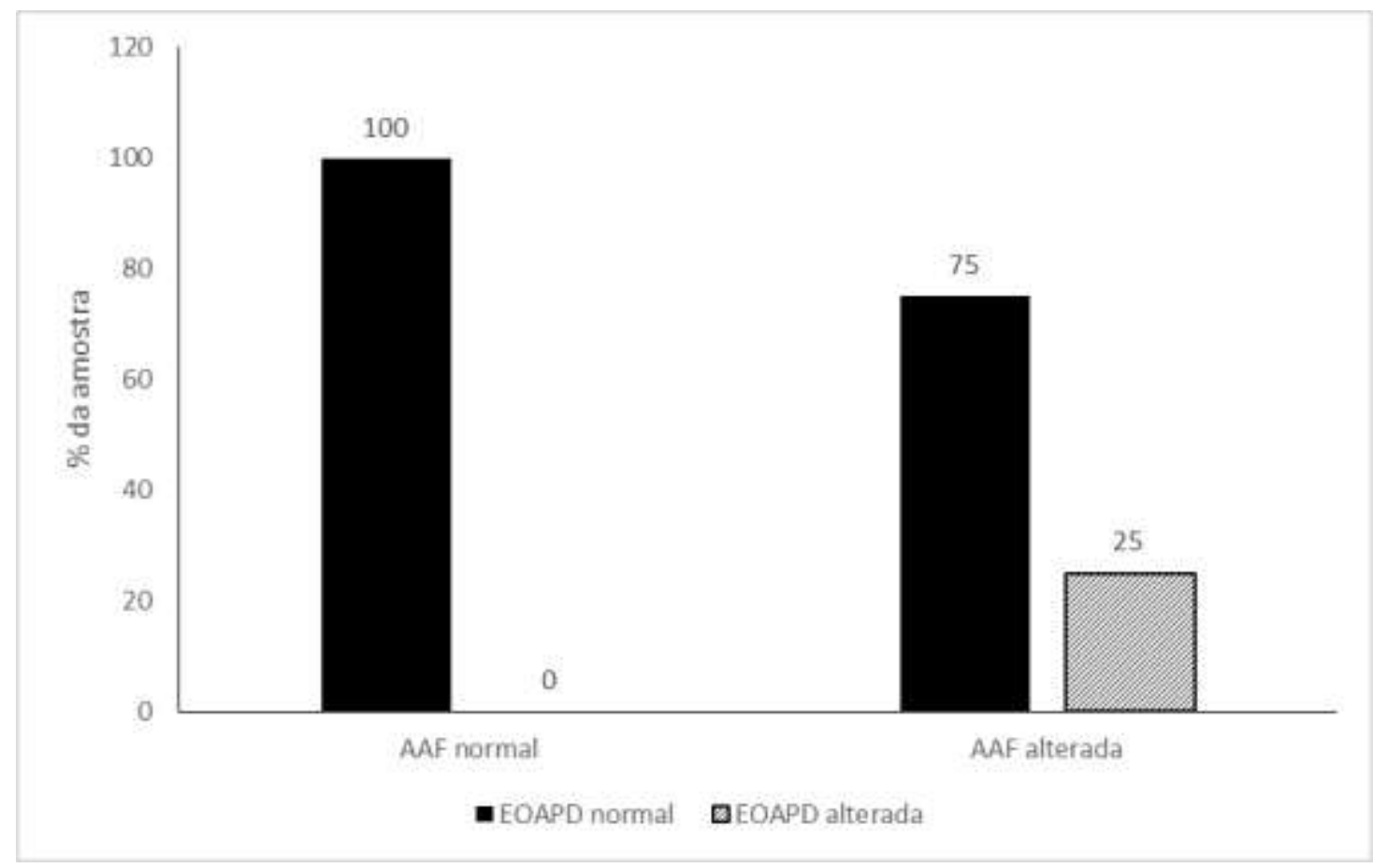

Legenda: \% - valores porcentuais; AAF - audiometria de altas frequências; EOAPD - emissões otoacústicas por produto de distorção. Fonte: autores.

Figura 4 - Associação entre os resultados das alterações das emissões otoacústicas por produto de distorção e a perda auditiva em altas frequências no Grupo 2.

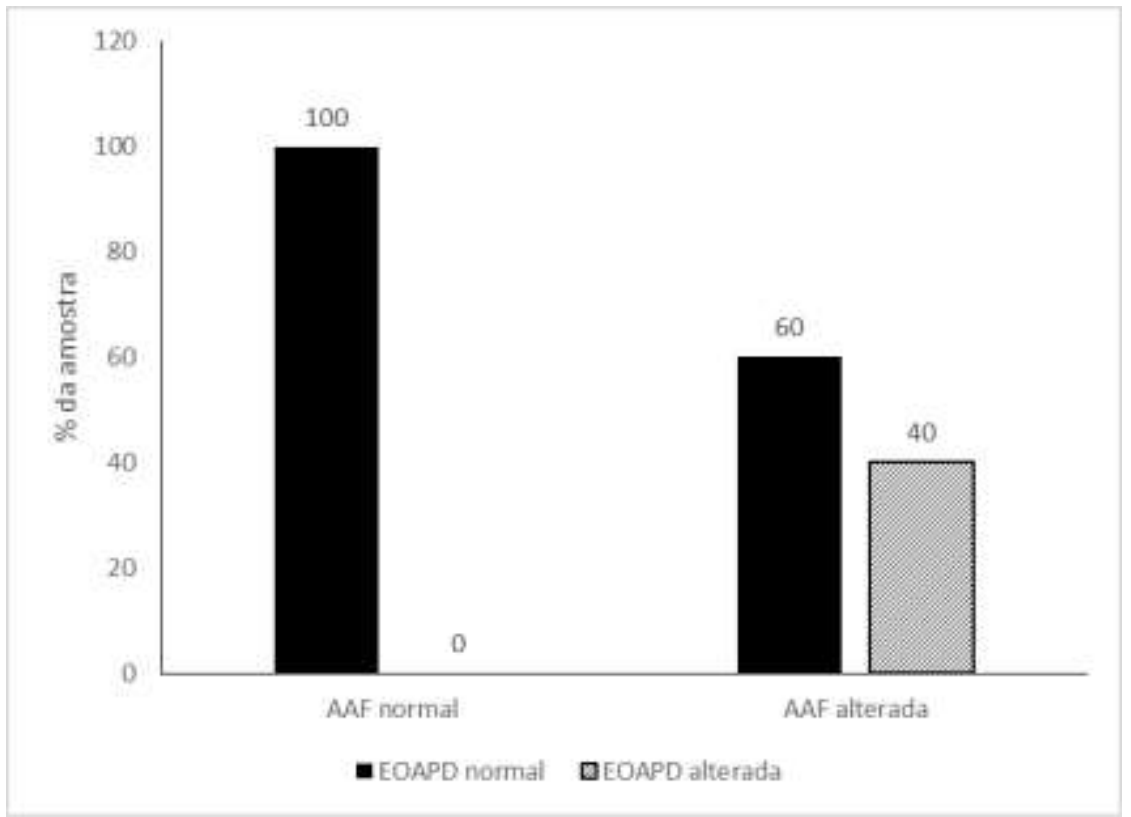

Legenda: \% - valores porcentuais; AAF - audiometria de altas frequências; EOAPD - emissões otoacústicas por produto de distorção. Fonte: autores. 
Quando comparadas a sensibilidade entre os dois exames (EOAPD e AAF) verificou-se diferença significativa somente no grupo $2(\mathrm{p}=0,004)$. No grupo 1 , apesar da diferença encontrada, o resultado não foi significativo ( $\mathrm{p}=0,250)$ (Figura $5)$.

Figura 5 - Comparação entre a sensibilidade dos dois exames nas crianças e adultos.

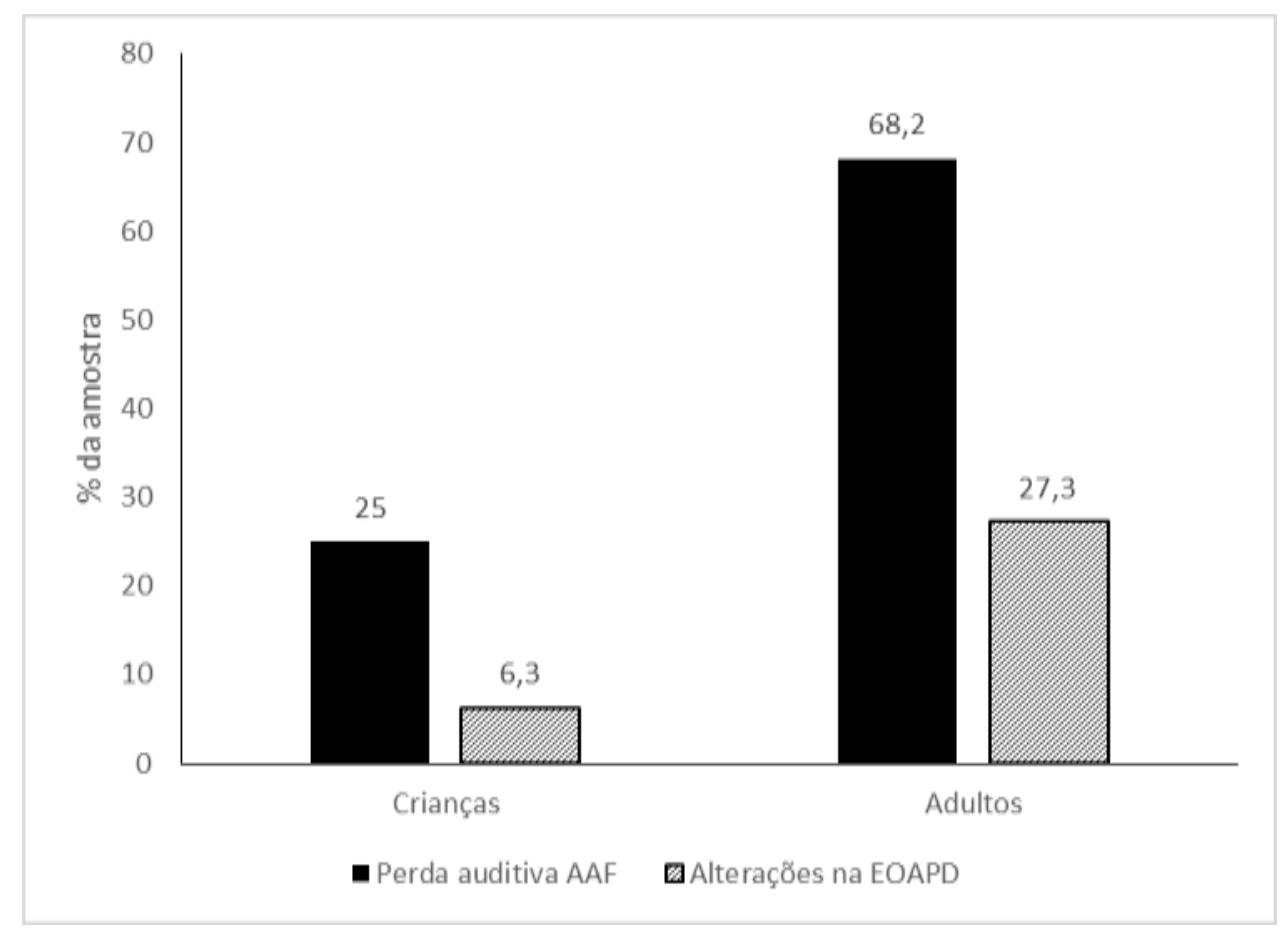

Legenda: \% - valores porcentuais; AAF - audiometria de altas frequências; EOAPD - emissões otoacústicas por produto de distorção. Fonte: autores.

\section{Discussão}

Com base nos resultados encontrados no presente estudo, pôde-se verificar que os indivíduos com mais de 20 anos de idade apresentaram maior porcentagem de perda auditiva nas frequências ultra-altas, em comparação aos mais novos. Nas frequências convencionais, também se verificou um percentual maior neste grupo, mesmo. Esse mesmo grupo apresentou valores aumentados, nos resultados das EOAPD, com diferença significativa na frequência de $2.800 \mathrm{~Hz}$ na orelha esquerda e $6.000 \mathrm{~Hz}$ na orelha direita, quando comparados com o grupo 1. Na associação das alterações das EOAPD com as frequências convencionais, houve significância ( $\mathrm{p}=0,013)$, ou seja, os pacientes adultos com alteração nas EOAPD também apresentaram perda auditiva nas frequências convencionais.

Os resultados supracitados são corroborados por estudo que realizou EOA transientes em crianças com AF, entre 6 meses e 10 anos de idade e não verificou diferença estatisticamente significante entre portadores de AF e grupo controle (Kegele et al., 2015). Outro estudo constatou apenas 6,6\% (12 crianças) com perda auditiva neurossensorial de um total de 181 com AF (Farrel, Landry, Yee, Leu \& Goudy, 2019).

Em contrapartida, uma outra pesquisa investigou patologias otológicas em crianças nigerianas com AF, comparando com grupo controle, encontrou um maior número de patologias, bem como maior prevalência de perda auditiva neurossensorial de grau leve no grupo com AF (Olajuyin, Olatunya, Adegbiji, Oyenibi \& Faboya, 2018). Assim como outro estudo que encontrou 28,8\% de perda auditiva neurossensorial em indivíduos entre 6 e 18 anos, com diferença estatisticamente 
significativa em comparação com o grupo controle (Lago et al., 2018) e outro que aponta maior prevalência de perda auditiva em crianças DF do que em crianças que não apresentam a doença (Stuart \& Press, 2012).

Recente trabalho que investigou os efeitos da AF na audição e equilíbrio de 46 indivíduos adultos, verificou que nas frequências entre $250 \mathrm{~Hz}$ e $16.000 \mathrm{~Hz}$, dos sujeitos com AF, os limiares auditivos foram significativamente maiores, em comparação ao grupo controle, de maneira que as frequências ultra-altas estavam mais prejudicadas que as convencionais, concordando com os resultados da presente pesquisa (Sarac, Boke \& Okuyucu, 2018). A espira basal da cóclea trata-se de uma região mais sensível à anóxia em função do alto consumo de oxigênio das estrias vasculares e também por ser uma estrutura com escassez de mecanismos anaeróbicos, o que pode justificar a perda auditiva nas frequências mais altas ((Olajuyin, Olatunya, Adegbiji, Oyenibi \& Faboya, 2018; Sarac, Boke \& Okuyucu, 2018).

Essa elevação dos limiares conforme o aumento da frequência, nos reporta à sensibilidade na detecção de alterações auditivas com a AAF, estando de acordo com estudo com pacientes com fibrose cística (Geyer, Menna Barreto, Weigert \& Teixeira, 2015). Outro estudo que fez uso do mesmo critério para avaliação das amplitudes (relação S/R) encontrou relação significativa entre a perda auditiva nas frequências mais altas (até $8.000 \mathrm{~Hz}$ ) e as EOAPD estavam presentes nas regiões de frequência onde a sensitividade auditiva para tons puros era normal. A amplitude das EOAPD estava reduzida quando os limiares estavam aumentados e ausentes nas frequências em que os limiares eram superiores a 50 dBNA para 251 das 255 orelhas testadas. A reavaliação pós-quelação indicou uma diminuição da amplitude ou ausência de EOAPD na presença de função da orelha média normal, mas essa diferença não foi estatisticamente significante. Esse estudo corrobora nossos achados, pois, em adultos, nossa associação de alteração em frequências convencionais e alteração das EOAPD também foi significativa ( $p=0,013$ ), embora não tenha sido informado no estudo a idade dos participantes (Burch-Sims \& Matlock, 2005).

Pesquisa realizada com 13 crianças com AF e 13 de grupo controle, não constatou diferença estatisticamente significativa nas EOA por estímulo transiente entre os grupos (Stuart \& Preast, 2012).

Estudos indicam que a lesão coclear ocorre devido às células falcêmicas que que interrompem o vasto suprimento sanguíneo necessário à elevada atividade metabólica requerida para manter o equilíbrio iônico e elétrico da endolinfa e a natureza altamente dependente de respostas evocadas a partir da orelha interna em breves episódios de anóxia provocaria um dano coclear extensivo e progressivo (Sarac, Boke \& Okuyucu, 2018), o que justificaria a ausência de emissões otoacústicas acústicas em indivíduos com anemia falciforme (Silva, Nova \& Lucena, 2012).

Kemp (2002) avaliou 80 sujeitos de oito a 20 anos de idade (praticamente com a mesma faixa de idade das crianças e adolescentes avaliados no presente estudo, 40 do grupo controle e 40 portadores de DF, onde nenhum dos participantes apresentaram curva tipo $\mathrm{B}$, como no presente estudo. Com relação às EOAPD, não encontraram diferença significativa entre sexo e orelhas. As EOAPD tiveram amplitude menor no grupo com DF. Por terem utilizado a média e DP dos achados, não foi possível comparar com nosso estudo, pois utilizou- se a mediana e intervalo interquartílico.

Na comparação entre as sensibilidades dos exames AAF e EOAPD, houve diferença significativa entre eles somente no grupo $2(\mathrm{p}=0,004)$. No grupo 1 , apesar da diferença encontrada, o resultado não foi significativo $(\mathrm{p}=0,250)$. Por outros estudos, temos conhecimento da precocidade na detecção de alterações auditivas com a AAF (Geyer, Menna Barreto, Weigert \& Teixeira, 2015).

Estudo com pacientes apresentando doença falciforme, que avaliou a audição nas frequências convencionais, PEATE, APAC e EOAPD, encontrou prevalência de perda auditiva de 0 a 66\% (Burch-Sims \& Matlock, 2005).

Verificam-se que os estudos com AF geralmente fazem uso de avaliações audiológicas com frequências convencionais, sendo que há escassez de trabalhos com AAF e emissões otoacústicas. Ainda, os resultados encontrados na literatura divergem no que diz respeito a presença de perda auditiva em diferentes faixas etárias, evidenciando a necessidade da realização de mais estudos englobando diferentes faixas etárias. 
A maioria dos estudos analisa a média e DP das amplitudes das EOA (Stuart \& Preast, 2012). Em função de nossa distribuição ter sido assimétrica, as análises foram realizadas com a mediana e intervalo interquartílico, impossibilitando assim, uma comparação com outros estudos para corroborar o achado.

\section{Conclusão}

Os resultados do presente estudo evidenciaram porcentagem maior de limiares elevados na audiometria de altas frequências no grupo de adultos. Além disso, os limiares de audiometria de altas frequências apresentaram-se mais elevados do que os limiares das frequências avaliadas na audiometria convencional. As medidas de relação sinal/ruído das EOAPD foram menores no grupo dos adultos e as alterações apresentaram valores maiores nas frequências de $2800 \mathrm{~Hz}$ na orelha esquerda e $6000 \mathrm{~Hz}$ na orelha direita.

Acredita-se que novas pesquisas devem ser realizadas, especialmente no que se refere a estudos longitudinais, que analisem de forma continuada os limiares auditivos em freqüências convencionais e altas frequências e as emissões otoacústicas, tanto nas crianças quanto nos adultos que apresentam anemia falciforme, pois sabe-se das limitações que existem em um estudo transversal, onde somente um momento da vida dos indivíduos é analisado.

\section{Referências}

Burch-Sims, G. P., \& Matlock, V. R. (2005). Hearing loss and auditory function in sickle cell disease. Journal of Communication Disorders, 38, 321-329.

Farrel, N. A., Landry, A. M., Yee, E., Leu, R. M., \& Goudy, S. L. (2019). Sensorineural hearing loss in children with sickle cell disease. International Journal of Pediatric Otorhinolaryngology, 118, 110-114.

Geyer, L. B., Menna Barreto, S. S., Weigert, L. L., \& Teixeira, A. R. (2015). High frequency hearing thresholds and product distortion otoacoustic emissions in cystic fibrosis patients. Brazilian Journal Otorhinolaryngology, 81(6), 589-597.

Kegele, J. et al. Otoacoustic emission testing in Ghanaian children with sickle cell disease. (2015). Tropical Medicine and International Health, 20 (9), 12091212 .

Kemp, D.T. (2002). Otoacoustic emissions, their origin in cochlear function, and use. British Medical Bulletim, 63(1), $223-41$.

Köche, J. C. (2011). Fundamentos da metodologia científica: teoria da ciência e iniciação à pesquisa: Editora Vozes.

Lago, M. R. R. et al. (2018). Sensorineural hearing loss in children with sicklecell anemia and its association with endotelial dysfunction. Hematology, 23(10), $848-855$.

Olajuyin, A. O., Olatunya, O. S., Adegbiji, A. W., Oyenibi, A. S., \& Faboya, A. O. (2018). Otological burdens of Nigerian children with sickle cell disease. International Journal of Pediatric Otorhinolaryngology, 107, 1-5.

Oliveira, N. et al. (2009). Oxigenoterapia hiperbárica no tratamento da surdez súbita idiopática. Revista Portuguesa de Otorrinolaringologia e Cirurgia Cérvico-Facial, 47(4), 197-202.

Potoka, K. P., \& Gladwin, M. T. (2015). Vasculopathy and pulmonary hypertension in sickle cell disease. American Journal of Physiology-Lung Cellular and Molecular Physiology, 308(4), L314-324.

Psaltakos, V. et al. (2013). Cochlear dysfunction. in. patients with acute hypothyroidism. European Archives of Otorhinolaryngology, $270(11), 2839-2848$.

Rodrigues, P. A. M. (2017). Surdez súbita e oxigenoterapia hiperbárica. Dissertação (Mestrado Integrado em Medicina), Universidade de Lisboa, Lisboa, Portugal.

Sarac, E. T., Boke, B., \& Okuyucu, S. (2018). Evaluation of Hearing and Balance Functions of Patients with Sickle Cell Anemia. Audiology and Neurotology, $23,122-125$.

Silva, L. P. A., Nova, C. V., \& Lucena, R. (2012). Anemia falciforme e surdez infanto-juvenil: revisão da literatura. Brazilian Journal of Otorhinolaryngology, 78(1), 126-131.

Silva, P. B., Fiorini, A. C., \& Azevedo, M. F. (2017). Estudo das emissões otoacústicas em indivíduos expostos a ruído de bateria universitária. Revista CEFAC, 19(5), 645-653.

Stuart, A., \& Preast, J. L. (2012). Contralateral suppression of transient-evoked otoacoustic emissions in children with sickle cell disease. Ear and Hearing, 33(3), 421-429. 
Research, Society and Development, v. 10, n. 4, e0510413627, 2021

(CC BY 4.0) | ISSN 2525-3409 | DOI: http://dx.doi.org/10.33448/rsd-v10i4.13627

Stuart, A., \& Smith, M. R. (2019). The emergence and prevalence of hearing loss in children with homozygous sickle cell disease. International Journal of Pediatric Otorhinolaryngology, 123, 69-74.

Weigert, L. L., Piltcher, O. B., Procianoy, E. F. A., Buss, C. H., \& Menna-Barreto, S. S. (2013). Avaliação do uso da audiometria em frequências ultra-altas em pacientes submetidos ao uso de ototóxicos. Brasília Medica, 50, 264-73. 